\title{
Distal myopathy, Tateyama type
}

INSERM

\section{Source}

INSERM. (1999). Orphanet: an online rare disease and orphan drug data base. Distal myopathy, Tateyama type. ORPHA:488650

Distal myopathy, Tateyama type is a rare, genetic, slowly progressive, distal myopathy disorder characterized by muscle atrophy and weakness limited to the small muscles of the hands and feet (in particular, thenar and hypothenar muscle atrophy), increased serum creatine kinase, and severely reduced caveolin-3 expression on muscle biopsy. Some patients may also show calf hypertrophy, pes cavus, and signs of muscle hyperexcitability. 